\title{
PRIMER REgISTRO DE LA MALEZA AGRícola E INVASIVA AMARANTHUS PALMERI (AMARANTHACEAE) PARA LA FLORA DE LA PENÍNSULA DE YUCATÁN Y UN REGISTRO ACTUALIZADO DE LA DIVERSIDAD DE AMARANTHUS EN LA REGIÓN
}

\section{FIRST RECORD OF INVASIVE AND AGRICULTURAL WEED AMARANTHUS PALMERI (Amaranthaceae) for the Peninsula of Yucatan Flora AND AN UPdATEd RECORd OF AMARANTHUS DIVERSITY IN THE REGION}

\author{
IVONNe SÁNCHEZ-DEL PINO ${ }^{1, *}$, Alfredo DORANTES-EUAN $^{1}$ y ARIADNA IBARRA-Morales ${ }^{1}$
}

${ }^{1}$ Centro de Investigación Científica de Yucatán A.C. Mérida, Yucatán, México.

*Autor para la correspondencia: isanchez@cicy.mx

\begin{abstract}
Resumen
Antecedentes: Amaranthus palmeri es una hierba dioica que se ha convertido en una especie invasora por su capacidad de adaptación y alta resistencia a herbicidas. Es considerada una de las especies más problemáticas para el sector agrícola de Estados Unidos de América, por lo que su documentación es de importancia para una región que alberga una alta biodiversidad y gran actividad agrícola, como la Península de Yucatán.

Problema: Amaranthus palmeri se distribuye en la Península de Yucatán.

Especie de estudio: Amaranthus palmeri S. Watson

Sitio de estudio: Dos localidades en Yucatán, México, en 2014, 2017 y 2018.

Métodos: Se examinaron, caracterizaron y midieron caracteres morfológicos de la especie y se ilustraron sus caracteres florales.

Resultados: Amaranthus palmeri es reportado por primera vez en la Península de Yucatán. Se describen los ejemplares colectados, discutiendo sus diferencias y similitudes con otras especies del género en la región. La variación intraespecífica de las poblaciones colectadas es documentada. Una clave actualizada para las especies de Amaranthus en la Península de Yucatán es incluida.

Conclusiones: Se identificaron 12 especies de Amaranthus distribuidas en la Península de Yucatán, incluyendo el registro de A. palmeri. La variación morfológica en los sépalos de las flores femeninas de A. palmeri podría indicar hibridación, plasticidad fenotípica o variación intraespecífica, los cuales pudieran estar relacionados con su capacidad de adaptación a los sistemas agrícolas y ambientes artificiales que coloniza, por lo que todo el conocimiento que se genere de esta especie puede ayudar a plantear soluciones más eficaces para su control.
\end{abstract}

Palabras clave: Amaranthus, amarantos, invasiva, maleza, morfología floral.

\begin{abstract}
Background: Amaranthus palmeri is a dioecious weed that has become an invasive species because of its proliferation ability and high resistance to herbicides. This species is considered one of the most troublesome weed species for the United States of America agricultural system, therefore, the documentation of this species is important for a region that harbors high biodiversity as well as many agricultural activities, as the Peninsula of Yucatan.

Question: Amaranthus palmeri is distributed in Peninsula of Yucatan.

Studied species: Amaranthus palmeri S. Watson

Study site: Two sites in Yucatán, Mexico in 2014, 2017 y 2018.

Methods: Morphological characters were examined, characterized and measured, and floral characters were photographed.

Results: Amaranthus palmeri is documented for the first time in the Peninsula of Yucatan. The collected specimens are described. Differences and similarities with other species of the genus in the region are discussed. Intraspecific variation of the populations is reported. A key to the species of Amaranthus in the Peninsula of Yucatan is provided.

Conclusions: Twelve species of Amaranthus were identified in the Yucatan Peninsula, including A. palmeri. The morphological variation of sepals of the female flowers of $A$. palmeri might indicate hybridization, phenotypic plasticity or intraspecific variation, which might be related to its ability to adapt to the agricultural systems and artificial environments that colonizes; therefore, the generation of knowledge for this species might be helpful in designing more efficient management solutions.
\end{abstract}

Key words: amaranths, Amaranthus, flower morphology, invasive, weed.

This is an open access article distributed under the terms of the Creative Commons Attribution License CCBY-NC (4.0) international. https://creativecommons.org/licences/by-nc/4.0/ 
El género Amaranthus L. (Amaranthaceae Juss.) tiene alrededor de 70 especies distribuidas en todo el mundo y alrededor de 40 especies son nativas de América (Hernández-Ledesma et al. 2015, Iamonico 2015b). El género incluye especies dioicas y monoicas. De las especies monoicas, tres especies son domesticadas y tienen importancia económica, i.e. A. caudatus L, A. cruentus L. y. A. hypochondriacus L (Sauer 1950), mientras que sólo existen diez especies dioicas en el género, entre las que se encuentra $A$. palmeri S.Watson (Sauer 1955).

Amaranthus palmeri es una hierba anual nativa de Norteamérica que taxonómicamente ha sido clasificada dentro del subgénero Acnida (L.) Aellen ex K.R. Robertson junto con el resto de los amarantos dioicos (Ward et al. 2013). Mosyakin \& Robertson (1966) propusieron la sección Saueranthus Mosyakin \& K. R. Robertson (tipo: A. palmeri) para incluir las especies dioicas de Amaranthus con cinco sépalos en flores pistiladas y frutos generalmente dehiscentes. Sin embargo, distintos estudios genéticos y morfológicos proporcionan evidencias que la ubican más cercanamente relacionada a la especie monoica Amaranthus spinosus L. (el quintonil espinoso; Franssen et al. 2001, Wassom \& Tranel 2005), con el que además hibrida frecuentemente (Ward et al. 2013). Amaranthus palmeri ha tenido una larga relación con el ser humano. Sauer (1955) destaca su importancia alimenticia al señalar que muchas tribus norteamericanas, como los Mojave, Chemehuevi y Papago de Arizona, los Cocopa de Sonora, los Tarahumara de Chihuahua, entre otros, la consumían como verdura o como grano. Recientemente, la especie ha cobrado relevancia al ser considerada una maleza que afecta la productividad de campos agrícolas (Culpepper et al. 2006, Norsworthy et al. 2008, Ward et al. 2013).

Las especies invasoras no son raras en el género $\mathrm{Ama}$ ranthus, ya que muchas de éstas se han dispersado más allá de sus propios campos de cultivo y se han convertido en un problema agrícola y biológico al competir con las especies nativas por nutrientes, generando pérdidas económicas y de biodiversidad (Iamonico 2015b, Iamonico \& El Mokni 2017, 2018). Todas las especies del subgénero Acnida son nativas de Norteamérica, pero se distribuyen en otras regiones como especies introducidas, algunas veces invasoras (Iamonico \& El Mokni 2017). Las plantas invasoras son aquellas naturalizadas o introducidas que consiguen establecerse y dispersarse a nuevos hábitats, sin ayuda directa del ser humano (Rejmánek 2000, Radosevich et al. 2007), lo cual representa una amenaza muy seria a la biodiversidad local (MüllerSchärer 2004).

La distribución de $A$. palmeri no es la excepción, aunque no fue cultivada sistemáticamente por los nativos de su área de distribución original (Sauer 1955), es posible que el transporte de semillas o el incremento de zonas agrícolas haya desencadenado su expansión territorial (Sauer 1955, Ward et al. 2013). A pesar de ser una especie dioica, su ventaja de colonización radica en su rápida capacidad de crecer y proliferar en ambientes artificiales (Sauer 1957). Esta capacidad adaptativa a ambientes hostiles y su resistencia a una gran cantidad de herbicidas (Culpepper et al. 2006, Norsworthy et al. 2008, Ward et al. 2013, Nakka et al. 2017) la han convertido en una de las especies más problemáticas y nocivas para el sector agrícola de Estados Unidos de América (EUA; Ward et al. 2013).

El control de Amaranthus palmeri en los campos de cultivo de EUA es complejo ya que, en las últimas tres décadas, ha desarrollado resistencia a distintos herbicidas, siendo aquellos de los que se ha documentado más información al respecto: la dinitroanilina (Gossett et al. 1992), la triazina (Jhala et al. 2014), los inhibidores de la acetolactato sintasa (Horak \& Peterson 1995, Gaeddert et al. 1997), los inhibidores de la 4-hidroxifenilpiruvato dioxigenasa (Jhala et al. 2014), los inhibidores de la protoporfirinógeno oxidasa (Heap 2019) y al glifosato (inhibidor de la EPSP sintasa; Culpepper et al. 2006). Este último, es uno de los herbicidas más utilizados en EUA (Bravo et al. 2018). También se han reportado plantas de $A$. palmeri con resistencia a algunos de estos herbicidas en Israel, Argentina y Brasil (Heap 2019).

Además de la resistencia a herbicidas, su invasión a los campos de cultivo se ve promovida y acelerada por prácticas agrícolas tales como la labranza, la cosecha y el composteo, ya que incluso estas actividades logran favorecer la dispersión de las semillas (Norsworthy et al. 2014). Su rápida proliferación le confiere a $A$. palmeri ventajas sobre plantas cultivadas de crecimiento lento como el algodón (Gossypium hirsutum L.), el maíz (Zea mays L.) y la soya (Glycine $\max$ (L.) Merr; Klingaman \& Oliver 1994, Rowland et al. 1999, Smith et al. 2000, Massinga et al. 2001, Morgan et al. 2001).

Dentro del continente americano esta especie ha sido reportada en lugares tan remotos como Argentina (Morichetti et al. 2013), y también se ha dispersado a otros continentes (Iamonico 2015a, Iamonico \& El Mokni 2017). En México se han reportado 33 especies de amarantos y una de las regiones con más alta diversidad comprende el noroeste de la Península de Yucatán (Espitia et al. 2010). En la Península de Yucatán se han registrado 11 especies de Amaranthus (Apéndice 1), entre las que se encuentran A. arenicola I.M. Johnst, A. australis (A.Gray) J.D. Sauer, A. crassipes Schltdl. var. crassipes, A. cruentus L., A. dubius Mart. ex Thell., A. greggii S. Watson, A. hybridus L., A. polygonoides L., A. powellii $\mathrm{S}$. Watson, A. spinosus y $A$. viridis L. (Sauer 1955, Standley \& Steyermark 1946, González-Iturbe et al. 2002, Sánchez-del Pino et al. 2013, Solís-Fernández 2017); de las cuales, tres son especies dioicas (A. arenicola, A. australis y $A$. greggi).

Las especies de Amaranthus tienen capacidad de hibridar con otras especies del género, aún entre especies monoicas y dioicas (Sauer 1957, Franssen et al. 2001, Ward et al. 2013), por lo que los límites de éstas pueden ser difíciles de definir y la variación morfológica es elevada. Se han encontrado híbridos silvestres, como cruzas entre $A$. hybridus $\times A$. cruentus o viceversa, y amarantos con morfología aberrante en la Península de Yucatán. La documentación de una especie invasora y nociva, con potencial daño económico y biológico, es de suma importancia para una región que alberga una alta biodiversidad y de gran actividad agrícola. En este estudio se reporta Amaranthus palmeri por primera vez en la Península 
de Yucatán, además se describen e ilustran sus principales caracteres florales con base en el material colectado y de herbario, y se incluye una clave con caracteres florales pistilados para las especies de Amaranthus reportadas para la región.

\section{Materiales y métodos}

La primera colecta de Amaranthus palmeri (L. Simá 8) resultó de una investigación denominada "conservación insitu y exsitu de la diversidad genética de amaranto" apoyada por COFUPRO (Coordinadora Nacional de las Fundaciones Produce A.C.) para ISP (Ivonne Sánchez-del Pino) con la actividad titulada "colecta de amaranto en el sur del país y su taxonomía del género" (clave BEI-AMA-13-3) que inició en 2014 y para el que se llevó a cabo un muestreo de amaranto en el sur del país, el cual incentivó la iniciativa de conocer el germoplasma de amarantos en el sureste mexicano. Tres años después, de manera independiente, se llevó a cabo la segunda colecta (A. Dorantes 713-718), la cual revivió el interés por identificar y caracterizar esta población de amarantos dioicos, que hasta ese momento solo se conocía en esa localidad. Una vez iniciado el proceso de caracterización e identificación taxonómica, se descubrió por casualidad al transitar en la vía pública una segunda población (A. Ibarra-Morales 100), la cual fue colectada e incluida en las mediciones y caracterización de la especie de la región.

Las muestras de flores fueron conservadas en FAA (Formaldehído-ácido acético-alcohol, Ruzin 1999). Las observaciones y fotografías se llevaron a cabo utilizando un microscopio estereoscópico Nikon SMZ745T con una cámara Lumenera Infinity 1. Los ejemplares fueron determinados con base en Sauer (1955) y Mosyakin \& Robertson (2004). Los ejemplares de respaldo fueron herborizados y depositados en CICY (Centro de Investigación Científica de Yucatán A.C., Thiers 2019+).

En las localidades muestreadas se colectaron plantas femeninas y masculinas de Amaranthus palmeri, y se presenta una descripción detallada de la morfología tanto de flores pistiladas como estaminadas. Sin embargo, la clave de identificación está elaborada principalmente con caracteres florales pistilados, ya que, de acuerdo con muchos autores (e.g. Sauer 1955, Carretero 1990, Akeroyd 1993, Costea et al. 2001, Bojian et al. 2003, Mosyakin \& Robertson 2004, Iamonico 2012, 2015b), estos caracteres son más confiables para la determinación de los amarantos dioicos y algunos trabajos recientes (e.g. Iamonico 2012, Adhikary \& Pratt 2015) han enfatizado su importancia taxonómica.

Además, en este trabajo se ilustra y describe la variación morfológica de flores pistiladas entre y dentro de las poblaciones colectadas en la región.

El estado migratorio de las especies indicadas en el Apéndice 1 se consideró evaluando la distribución nativa reportada por Eliasson (1987), Kauffman \& Weber (1990), Mosyakin \& Robertson (2004), Villaseñor \& Espinosa-García (2004), Vibrans (2009), Carmona-Pinto \& Orsini-Velásquez (2010), Iamonico (2015b) y Flores-Olvera et al. (2016). En este trabajo solo se consideraron dos estados: nativa e introducida.

\section{Resultados}

Amaranthus palmeri S. Watson (Figuras 1 y 2)

Plantas herbáceas, anuales, dioicas, hasta $160 \mathrm{~cm}$ de altura. Tallos erectos, ramificados, glabros, color verde a rojo. Hojas 1.5-7.2 $\times 0.7-3.3 \mathrm{~cm}$, lámina plana, oblanceoladas, lanceoladas a elípticas, base acuminada a cuneada, márgenes enteros, ápice acuminado a agudo, usualmente con mucrón terminal, glabras, venación prominente y blanquecina en la cara abaxial; pecíolos 0.4-6 cm de longitud. Inflorecencias terminales, tipo espiga o panícula, erectas, hasta $40.1 \mathrm{~cm}$ de largo y $1.5 \mathrm{~cm}$ de ancho. Flor pistilada $2.3-4.3 \mathrm{~mm}$ de largo. Bráctea 2.3-4.5 × 0.6-1.4 mm, nervadura central largamente excurrente y engrosada, igual o más larga que los sépalos externos, ápice largamente acuminado. Sépalos cinco, desiguales, lineales, espatulados u obovados, glabros o con pubescencia pilosa, ápice de la lámina obtuso, obcordado o agudo, vena media no sobresale de la lámina o excurrente con ápice mucronulado a pungente, vena media no ramificada o con ramificaciones secundarias, con margen entero, crenado o serrado rodeando el ápice y entero hacia la base del sépalo; sépalo externo 1.4-3.7 × 0.4-1.2 $\mathrm{mm}$; sépalos internos 0.8-2.5 $\times 0.3-1.1 \mathrm{~mm}$; estigmas 2(-3), 0.69-2.58 mm de largo. Ovario ovoide, $0.31-0.9 \times 0.22-0.56 \mathrm{~mm}$. Utrículo obovoide, 1.1-2 $\times$ 0.7-1.7 mm con línea de dehiscencia, parte superior del utrículo 0.41-0.82 mm de largo, muy rugoso y con apéndices semicirculares de hasta $0.22 \mathrm{~mm}$ de ancho que sobresalen del margen del utrículo justo por encima de la línea de dehiscencia, parte inferior del utrículo 0.43-1.01 mm de largo, liso a ligeramente rugoso. Semillas redondas a ligeramente obovadas, lenticulares, color pardo rojizo oscuro, brillantes, 0.7-1.4 mm diámetro. Flor estaminada $2.4-4.4 \mathrm{~mm}$ de largo. Bráctea 1.1-3.5 × 0.5-1 mm, con nervadura central largamente excurrente y engrosada, igual o más larga que los sépalos externos, ápice largamente acuminado. Sépalos cinco, desiguales, ovalados a oblongos, ápice agudo, margen entero; sépalo externo $2.2-4.2 \times 0.6-1 \mathrm{~mm}$, con nervadura central largamente excurrente; sépalos internos 1.7-2.9 $\times 0.5-1 \mathrm{~mm}$, con nervadura central ligeramente excurrente. Estambres cinco, filamento 0.6-1.1 mm de largo, ausencia de apéndices en el androceo, anteras hasta $1.7 \mathrm{~mm}$ de longitud.

Ejemplares examinados. México, Yucatán, Parque Kai Lu'um, 9 msnm, 210 02' 31.74" N, 89³9' 14.14" O, 9.XI.2014, L. Simá 8; loc. cit., 22.IX.2017, A. Dorantes 713-718; loc. cit., 11.VII.2018, I. Sánchez-del Pino 588 y 589. Frente a la Secretaría de Seguridad Pública, 21 02' 16.8" N 89 37' 56.3" O, 07.IX.2018, A. Ibarra-Morales 100.

Observaciones de las poblaciones colectadas. La primera población de Amaranthus palmeri fue encontrada en un parque administrado por las autoridades municipales de la ciudad de Mérida, denominado Kai Lu'um ("El canto de la tierra" en lengua maya). El parque fue durante muchos años el basurero municipal de Mérida hasta que en 2013 se llevaron a cabo trabajos de rehabilitación para hacer de este lugar un sitio propicio para actividades recreativas familiares. Existe un cuerpo de agua permanente cuya área se incrementa en 

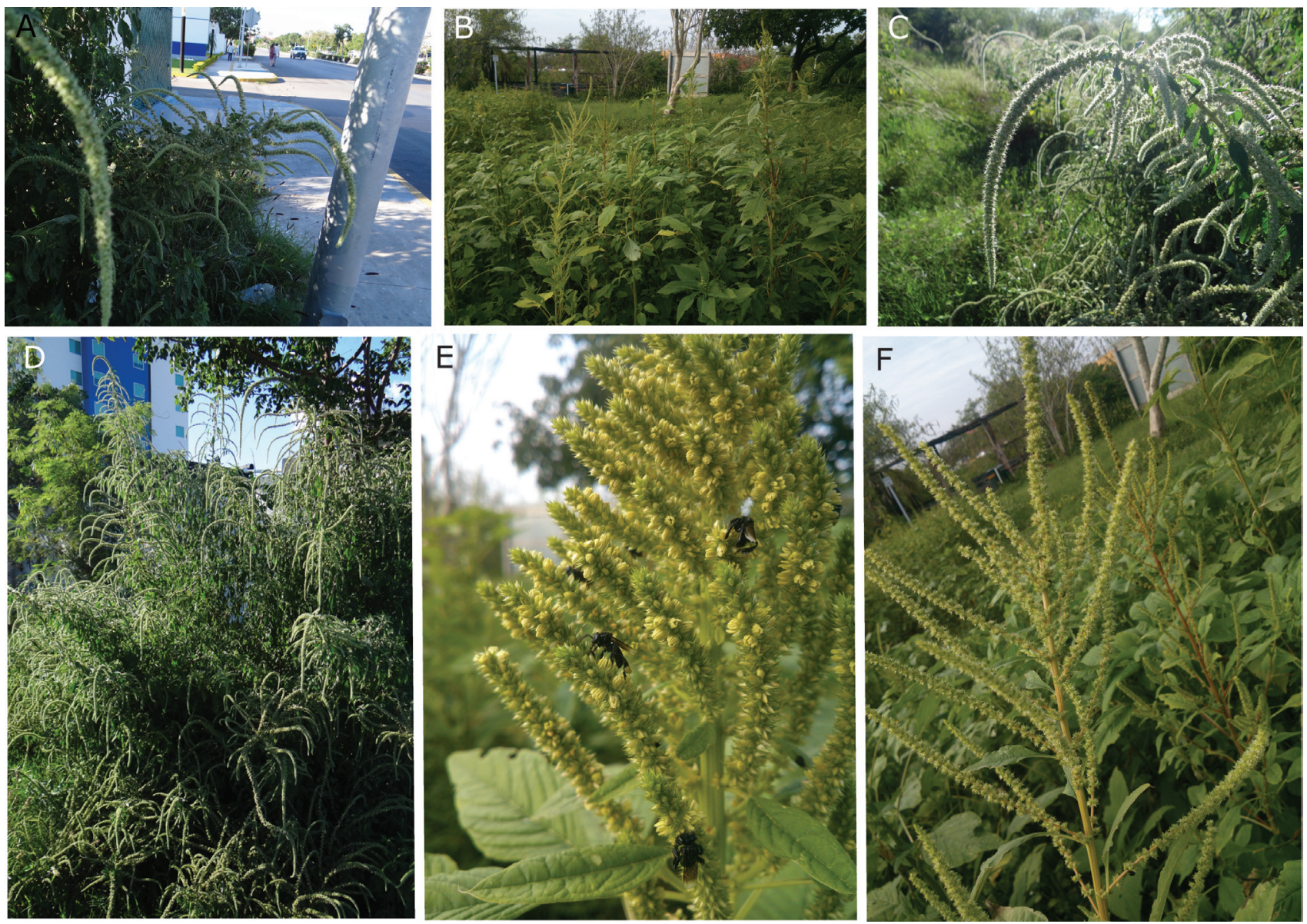

Figura 1. Amaranthus palmeri. A-F. Plantas en campo. A,C,D. Población de la colecta A. Ibarra-Morales 100. B,E,F. Población de la colecta A. Dorantes 713-718. E. Inflorescencia de flores masculinas. F. Inflorescencia de flores femeninas.

la época de lluvias, aunque en los últimos años la porción de agua ha disminuido considerablemente. En el parque se llevaron a cabo trabajos de reforestación principalmente con árboles y arbustos, los cuáles en su mayoría han sobrevivido y actualmente muchos son individuos adultos. Sin embargo, el porcentaje de cobertura por árboles y arbustos actualmente es bajo. Durante la época de lluvia la cobertura de vegetación se incrementa considerablemente, siendo el componente herbáceo muy importante. Entre las especies más abundantes en esta temporada se encuentran Amaranthus spp., Ipomoea spp., Merremia spp., Astraea lobata, Tithonia diversifolia, entre otras. La población de $A$. palmeri en este parque ocupa una superficie de aproximadamente $10,000 \mathrm{~m}$ cuadrados $(100 \times 100 \mathrm{~m})$, distribuyéndose en dos áreas contiguas, un área de aproximadamente 20 por 20 metros y otra área más grande de aproximadamente 175 por 55 metros, que eran atravesadas por senderos que son empleados para caminar a lo largo y ancho del parque. Esta especie se distribuye en el parque por lo menos desde 2014. En abril de 2018 se registró un incendio en el parque que abarcó un área considerable en donde crece $A$. palmeri, cabe mencionar que en esta época no existían individuos de dicha especie. Sin embargo, durante la época de lluvias las plantas de A. palmeri crecieron abundantemente, sin disminución perceptible del área que ocupaban en años anteriores.

La población correspondiente a la colecta A. IbarraMorales 100 se encontró por primera vez a principios de septiembre de 2018 creciendo en un terreno baldío, plantas masculinas y femeninas creciendo agregadas en la esquina suroccidental (que hace frente tanto a la Secretaría de Seguridad Pública como al estacionamiento de la Plaza Galerías), por lo menos 50 individuos con inflorescencias fueron observados. El sitio es un terreno sin construir con algo de basura en su lado occidental y sur, en las áreas cercanas a la vía pública. El mes siguiente se observó que una planta masculina en floración se encontraba creciendo en un camellón muy cercano a este sitio. En diciembre se observó una nueva población a $600 \mathrm{~m}$ de distancia sobre la misma vía, creciendo también en un terreno baldío. Esta última población, ya contaba con alrededor de 10 individuos con inflorescencias, por lo que no hay certeza acerca del momento de la colonización de la especie en este nuevo predio. 


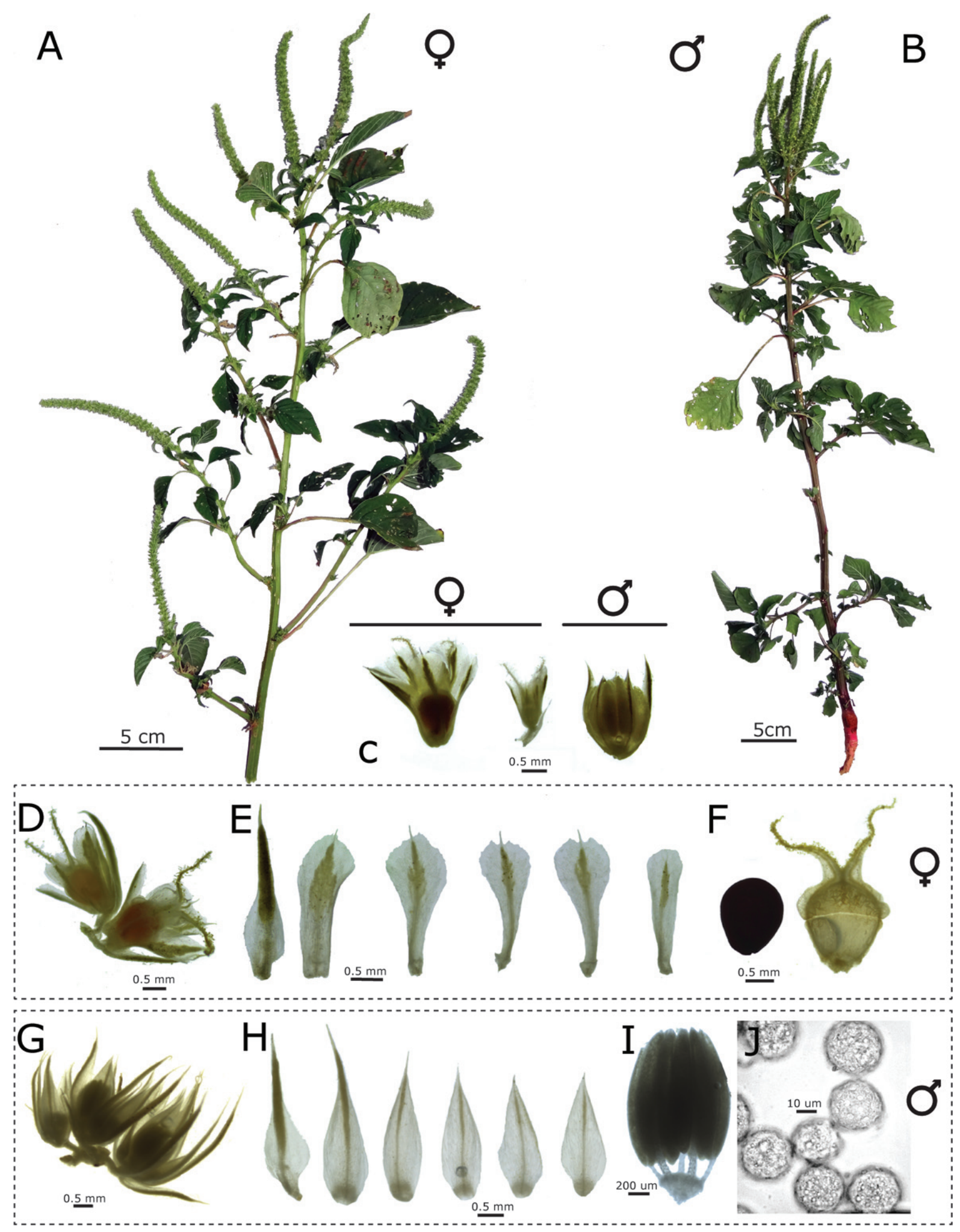

Figura 2. A. Planta femenina de A. palmeri. B. Planta masculina de A. palmeri. C. Flores femeninas y masculinas. Fruto, flor femenina juvenil y flor masculina (de izquierda a derecha). D-F. Estructuras de las flores femeninas. D. Espiga con dos flores femeninas con fruto inmaduro. E. Bráctea y cinco sépalos. F. Semilla y utrículo. G-I. Estructuras de las flores masculinas. G. Espiga con flores masculinas. H. Bráctea y cinco sépalos. I. Estambres. J. Polen. 
Distribución y ecología. La especie está reportada en México para los estados de Aguascalientes, Baja California, Baja California Sur, Chihuahua, Ciudad de México, Coahuila, Colima, Durango, Estado de México, Guerrero, Jalisco, Michoacán, Morelos, Nayarit, Nuevo León, Oaxaca, Puebla, San Luis Potosí, Sinaloa, Sonora, Tamaulipas, Veracruz y Zacatecas (Villaseñor \& Espinosa-García 1998, Sánchezdel Pino et al. 1999, Rzedowski \& Rzedowski 2005). Se han reportado colectas en selva baja caducifolia, bosque de Quercus sp., en suelo arenoso, fluvisol y arvense (Sánchezdel Pino et al. 1999), en localidades que van de los 0 a los 2092 msnm (Espitia et al. 2010). Sauer (1955) menciona que crece a lo largo de arroyos permanentes o intermitentes, en esos lugares crece sobre limo, suelos arenosos y gravosos, y añade que probablemente es el único de los amarantos dioicos cuyas colectas en ambientes artificiales superan al número de colectas en ambientes naturales. En la Península de Yucatán se encontró en la ciudad de Mérida, creciendo a 9 msnm en ambientes perturbados.

Variación morfológica. Las flores femeninas de Amaranthus palmeri presentaron una gran variación morfológica en la forma de los sépalos, particularmente en tamaño, margen y ápices (Figura 3). El ancho de los sépalos varió de 0.4-1.2 $\mathrm{mm}$ en los externos y de 0.3-1.1 en los internos. Se encontraron lineares, espatulados y obovados. Adicionalmente, la forma del margen en la región apical de los sépalos varió entre entero, crenado y dentado. El ápice en la lámina también varió entre obtuso, obcordado y agudo en los sépalos externos, mientras que en los sépalos internos fue obtuso y obcordado y la vena media fue excurrente en todos los sépalos externos, su ápice varió de mucronulado a pungente, y en los sépalos internos de ausente a apiculado.

En las plantas de la población colectada por $A$. IbarraMorales 100 se observaron flores pistiladas con diferencias morfológicas, las cuales se pueden separar en dos grupos (Figura 4, Tabla 1). Un grupo de flores presentó sépalos de dimensiones grandes y corresponde a la forma más frecuente. Por lo tanto, estas flores fueron aquí denominadas como flores comunes (Figura 4C). El segundo grupo de flores presentaron sépalos muy reducidos (Figura 4D) y estas flores fueron denominadas en este trabajo como flores inusuales. A continuación, se presenta una clave de identificación de las especies de Amaranthus que se encuentran en la Península de Yucatán.

Clave para las especies de Amaranthus en la Península de Yucatán

1a. Plantas dioicas . . . . . . . . . . . . . . . . . . . 2

1b. Plantas monoicas . . . . . . . . . . . . . 5

2a. Sépalos ausentes o poco desarrollados (de menos de 1 mm y sin vena media evidente) ............ australis* 2b. Sépalos presentes y bien desarrollados (de más de $1 \mathrm{~mm}$ y con vena media evidente).............. 3 3a. Fruto indehiscente ............... greggii* 3b. Fruto dehiscente. . . . . . . . . . . . . . . 4 4a. Plantas de hasta $50 \mathrm{~cm}$ de altura; hojas oblanceoladas o linear-lanceoladas de $0.3-0.5 \mathrm{~cm}$ de ancho; sépalo externo
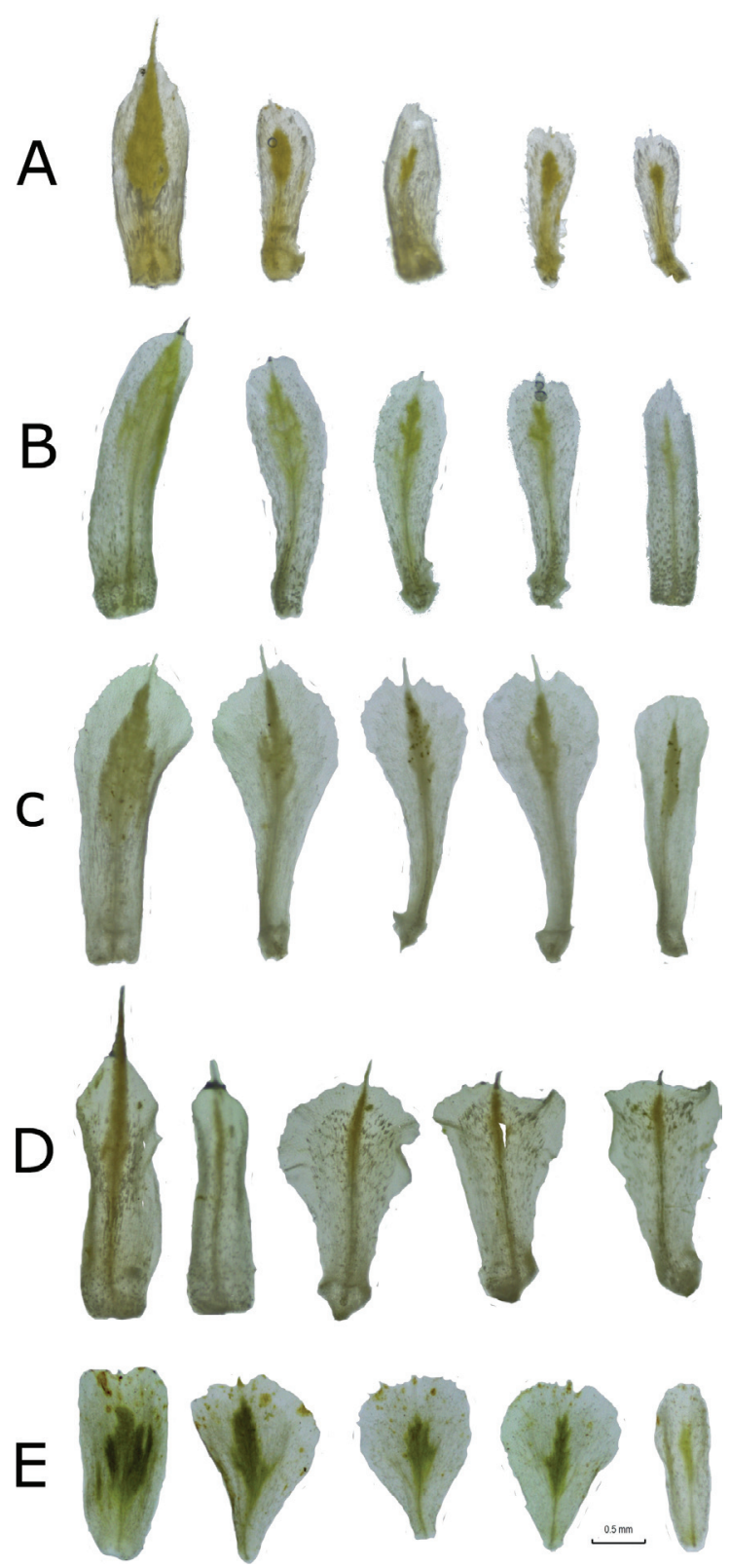

Figura 3. Variación en sépalos de flores femeninas de A. palmeri. A. Sépalos lineales a lanceolados con vena media ligeramente excurrente (A. Dorantes 713). B. Sépalos espatulados con vena media ligeramente excurrente (A. Ibarra-Morales 100). C-D. Sépalos espatulados a obovados con vena media largamente excurrente (I. Sánchez-del Pino 588 ). E. Sépalos obovados y lineales con vena media no excurrente o ligeramente excurrente (L. Simá 8).

obtuso o retuso, con vena media no excurrente más allá de la lámina, generalmente tres estigmas (a veces cuatro) .... . $\ldots \ldots \ldots \ldots \ldots \ldots \ldots \ldots$. arenicola* 4b. Plantas de hasta $1.60 \mathrm{~cm}$ de altura; hojas oblanceoladas, lanceoladas a elípticas de 0.7-3.3 cm de ancho; sépalo externo obtuso, obcordado o agudo, con vena media no excurrente 


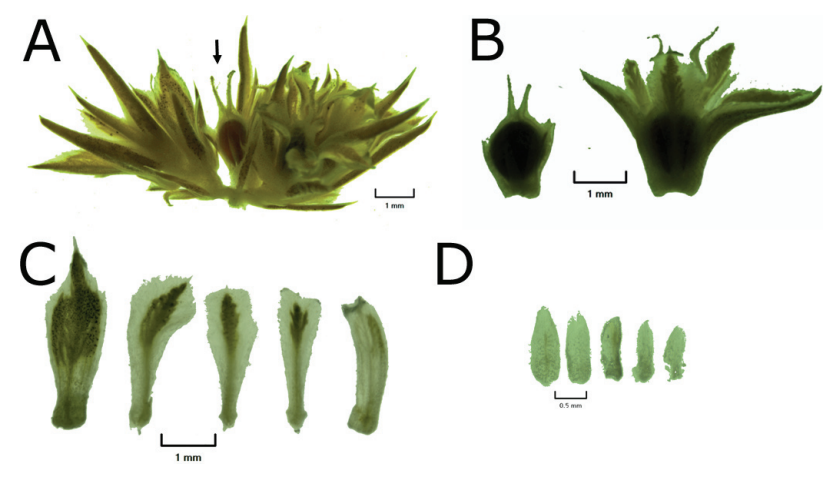

Figura 4. Flores pistiladas con morfología común e inusual ( $A$. Ibarra-Morales 100). A. Inflorescencia con flores comunes y flor inusual (flecha). B. Flor inusual y flor común. C. Sépalos de flor común. D. Sépalos de flor inusual.

a excurrente más allá de la lámina y entonces con ápice mucronulado a pungente, generalmente dos estigmas (rara vez tres $\ldots \ldots \ldots \ldots \ldots \ldots \ldots \ldots$. palmeri 5a. Inflorescencias axilares sostenidas por una espina (bráctea modificada) . . . . . . . . . . . . A spinosus ${ }^{*}$ 5b. Inflorescencias axilares no sostenidas por una espina . 6 6a. Fruto indehiscente . . . . . . . . . . . . . 7 6b. Fruto dehiscente. . . . . . . . . . . . . . 9 7a. Flores pistiladas obcónicas; sépalos de las flores pistiladas generalmente 3 (hasta 5), libres en la base, ampliamente obovados a lanceolados ................. viridis* 7b. Flores pistiladas urceoladas; sépalos de las flores pistiladas 5 , connados o fusionados en la base, espatulados . . . 8 8 8a. Inflorescencias cuyas ramas son rectas; estambres 2-3, semilla $<1 \mathrm{~mm}$ de diámetro ........... polygonoides* 8 b. Inflorescencia cuyas ramas son sinuosas-recurvadas; estambres 5 , semilla $\geq 1 \mathrm{~mm}$ de diámetro. ........... ................... crassipes var. crassipes* 9a. Brácteas de igual tamaño a más largas que las flores; sépalos de las flores femeninas igual a más largos que el

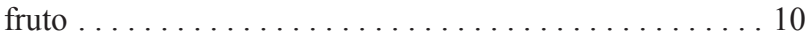
9b. Brácteas igual o de menor tamaño que las flores; sépalos de las flores femeninas ligeramente igual a más cortos que el fruto ...................... dubius* 10a. Brácteas acuminadas, más de dos veces (y hasta cuatro veces) más largas que los sépalos; sépalos de las flores pistiladas acuminados. ............... powellii* 10b. Brácteas agudas, hasta dos veces más largas que los sépalos; sépalos de las flores pistiladas agudos . . . . . . 11 11a. Sépalos de las flores femeninas más largos que el fruto; inflorescencias comúnmente verdosas ........ hybridus* 11b. Sépalos de las flores femeninas igual o más cortos que el fruto; inflorescencias comúnmente de color rojo . . . . . . .

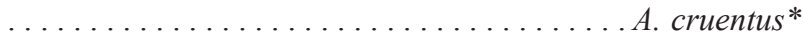
*Literatura recomendada con ilustraciones de estas especies se indican en el Apéndice 2.

\section{Discusión}

Amaranthus palmeri es una hierba dioica. Las plantas femeninas y masculinas presentan cinco sépalos desiguales, uno externo de mayor dimensión y cuatro internos más pequeños, así como una bráctea asociada a la flor. Esta especie podría ser confundida con $A$. powellii por compartir la presencia de brácteas con ápice laminar muy acuminado. La gran diferencia es que $A$. powellii es una especie monoica. Con respecto a las especies dioicas $A$. greggii y $A$. arenicola, el tamaño de la planta (de hasta $80 \mathrm{~cm}$ de alto en $A$. greggii y hasta $50 \mathrm{~cm}$ de alto en $A$. arenicola), la dehiscencia del fruto (indehiscente en $A$. greggii) y el número de estigmas (tres en $A$. arenicola y $A$. greggii) son caracteres que permiten diferenciarlas de A. palmeri.

La especie con una mayor similitud a $A$. palmeri es $A$. watsonii Standl., la cual aún no ha sido reportada para la Península de Yucatán, pero se distribuye en el norte de México, en los estados de Baja California, Baja California Sur, Sonora y Sinaloa (Sauer 1955, Sánchez-del Pino et al. 1999). Espitia et al. (2010) la ubica como la sexta especie de Amaranthus con mayor representación en México, con 96 colectas entre herbarios y bancos de germoplasma. La principal diferencia entre $A$. palmeri y $A$. watsonii es la pubescencia glandular que se presenta en el lado externo de la bráctea de $A$. watsonii (Sauer 1955, Mosyakin \& Robertson 2004), ausente en $A$. palmeri. Además, las diferencia el tamaño de los ápices de brácteas y sépalos de las flores pistiladas, que son más largos en $A$ palmeri que en $A$. watsonii (Sauer 1955, Sánchez-del Pino et al. 1999).

Las plantas de Amaranthus palmeri colectadas en la Península de Yucatán presentaron dimensiones que coinciden con las reportadas para la especie (Sauer 1955, Mosyakin \& Robertson 2004, Morichetti et al. 2013, Iamonico 2015b) con excepción de la longitud de las brácteas florales, cuyas dimensiones reportadas van de 2.5-6.3 mm de longitud (Sauer 1955, Sánchez-del Pino et al. 1999, Mosyakin \& Robertson 2004, Morichetti et al. 2013, Iamonico 2015b), mientras que las plantas examinadas en este estudio presentaron menores dimensiones (1.1-3.7 $\mathrm{mm})$, lo que extendería el rango de variación de este carácter.

En cuanto a la variación en la forma de los sépalos de las

Tabla 1. Medidas de los principales caracteres de florales pistilados de la colecta A. Ibarra-Morales 100 en flores comunes e inusuales.

\begin{tabular}{lcccccc}
\hline & $\begin{array}{c}\text { Bráctea } \\
(\mathrm{mm})\end{array}$ & $\begin{array}{c}\text { Sépalo externo } \\
(\mathrm{mm})\end{array}$ & $\begin{array}{c}\text { Sépalos internos } \\
(\mathrm{mm})\end{array}$ & $\begin{array}{c}\text { No. de } \\
\text { estigmas }\end{array}$ & $\begin{array}{c}\text { Utrículo } \\
(\mathrm{mm})\end{array}$ & $\begin{array}{c}\text { Semilla } \\
(\mathrm{mm})\end{array}$ \\
\hline Flores comunes & $2.35-3.49 \times 0.7-1.13$ & $2.02-3.66 \times 0.63-1.18$ & $1.31-2.54 \times 0.42-0.84$ & 2 & $1.2-1.58 \times 1.04-1.25$ & $0.99-1.31 \times 0.96-1.12$ \\
Flores inusuales & $2.6-2.79 \times 0.83-0.95$ & $1.16-1.2 \times 0.35-0.43$ & $0.76-1.02 \times 0.15-0.34$ & 2 & $1.2-1.55 \times 0.88-1.21$ & $0.81-1.11 \times 0.78-1.03$ \\
\hline
\end{tabular}


flores femeninas, tratamientos taxonómicos previos describen estos sépalos como espatulados, obovados o rómbico-lanceolados y su margen como crenulado u ocasionalmente denticulado (Standley 1917, Sauer 1955, Mosyakin \& Robertson 2004, Morichetti et al. 2013, Iamonico 2015b). A diferencia de los resultados de este estudio, la mayoría de los tratamientos no reportaron amplia variación en los caracteres y solo incluyeron una o dos de las características antes mencionadas. Por ejemplo, Standley (1917) los describe de angostamente espatulados a ampliamente espatulados con margen ocasionalmente denticulado; Iamonico (2015b) los describe obovado-espatulados; y Morichetti et al. (2013) los describen como espatulados, por lo que este trabajo sería el primero en reportar una amplia variación intra e interpoblacional en caracteres de flores femeninas dentro de esta especie, como se ha indicado en el apartado de variación morfológica de este texto. La elevada variación morfológica en las flores femeninas de $A$. palmeri, principalmente en los caracteres de sépalos, entre individuos aún de la misma localidad, podría tener diversos orígenes. Esta variación podría ser resultado de eventos de hibridación, los cuales son comunes en varias especies del género (Sauer 1950,1955) y han sido reportados en A. palmeri (Sauer 1957, Gaines et al. 2011, Ward et al. 2013). Una elevada variación intraespecífica o una elevada plasticidad fenotípica son otras posibles causas.

Chandi et al. (2013) encontraron una elevada variación genética en poblaciones de $A$. palmeri de Carolina del Norte y Georgia (EUA), que pudiera estar relacionada con la gran variación fenotípica asociada al crecimiento y desarrollo de la planta, así como a la resistencia a herbicidas en poblaciones de $A$. palmeri. Según los autores, elevados niveles de variabilidad genética permiten a las poblaciones adaptarse rápidamente a dinámicas condiciones ambientales debido a que hay una gran disponibilidad inmediata de alelos ventajosos. Partiendo de este supuesto, nosotros consideramos que elevados niveles de variabilidad genética podrían ser responsables de la variación morfológica aquí documentada, por lo que la variación observada podría estar relacionada con su rápida capacidad de adaptación a los sistemas agrícolas y ambientes artificiales que coloniza.

En la Península de Yucatán se identificaron 12 especies de Amaranthus, siendo una región de alta diversidad para el género, con un nuevo registro correspondiente a la presencia de $A$. palmeri en la región. La especie tan solo había sido reportada para los estados mexicanos del norte con máxima distribución al sur en Veracruz (Villaseñor \& EspinosaGarcía 1998, Sánchez-del Pino et al. 1999, Rzedowski \& Rzedowski 2005). Mediante la revisión de la base de datos abiertos de la UNAM, se encontraron dos ejemplares de herbario pertenecientes a Amaranthus palmeri provenientes de la Península de Yucatán. El ejemplar E. Cabrera \& H. de Cabrera 13829 (MEXU1367362!, imagen disponible en http://datosabiertos.unam.mx/IBUNAM:MEXU:1367362) colectado en Yucatán (México) y el ejemplar Juarez Palmillas 658 (MEXU1124145!, imagen disponible en http://datosabiertos.unam.mx/IBUNAM:MEXU:1124145) colectado en Campeche (México). Con base en esta información, podemos sugerir que Amaranthus palmeri ha estado en la Península de Yucatán desde por lo menos 1987. Las localidades en las que se ha colectado $A$. palmeri dentro de la región coinciden con los hábitats donde se ha colectado en el resto del mundo, es decir, que en general crece en lugares perturbados por el hombre, como lotes baldíos, campos de cultivo, a la orilla de carreteras y caminos, entre otros (Sauer 1955, Morichetti et al. 2013, Iamonico 2015b).

Es una especie común en Estados Unidos y ha sido reportada en Argentina y otros países de América del Sur (Morichetti et al. 2013, Gonçalves-Netto et al. 2016). En Estados Unidos ha sido considerada una de las malezas agrícolas más nocivas para la soya, el maíz y el algodón, donde se ha reportado que puede reducir el rendimiento de los cultivos en más del $60 \%$ de producción (Klingaman \& Oliver 1994, Rowland et al. 1999). Además, la especie ha desarrollado resistencia a distintos tipos de herbicidas (Culpepper et al. 2006, Norsworthy et al. 2008, Neve et al. 2011, Nakka et al. 2017).

El maíz de grano blanco ocupa el primer lugar de cultivo en superficie sembrada con más de 300 hectáreas en la Península de Yucatán, y también se cultiva soya a menor escala en los tres estados que la conforman (INEGI 2017a, 2017 b 2017c). Estos dos cultivos son potencialmente susceptibles a ser invadidos por Amaranthus palmeri, lo cual podría generar pérdidas económicas y promover el desarrollo de poblaciones resistentes a herbicidas en la región si no se planean estrategias para su control. El rápido desarrollo de resistencia a distintos herbicidas, así como la capacidad de adaptarse a distintos sistemas de cultivo, como la alternancia de cultivos, han llevado a algunas poblaciones de $A$. palmeri a aumentar su tamaño y su capacidad reproductiva (Bravo et al. 2017, 2018). Esto ha impedido que exista una propuesta de control unificada y efectiva, ya que los mecanismos de resistencia y los efectos de los distintos sistemas de cultivo aún se encuentran siendo estudiados (Franssen et al. 2001, Gaines et al. 2011, Bravo et al. 2017, 2018, Koo et al. 2018). Por lo anterior, todo el conocimiento que se genere de esta especie puede ayudar a plantear soluciones más eficaces para su control.

\section{Agradecimientos}

Agradecemos a Erick Aguilera-Cauich (CICY) y Luis Simá (CICY) por apoyo en la colecta de Amarantos en la Península de Yucatán. Nuestra gratitud a dos revisores anónimos por las sugerencias recibidas en el mejoramiento de este documento. También, agradecemos enormemente el apoyo recibido por COFUPRO clave BEI-AMA-13-3 y Ciencia Básica-Consejo Nacional de Ciencia y Tecnología (CONACYT) 2014 con número de proyecto 243153.

\section{Literatura citada}

Adhikary D, Pratt DB. 2015. Morphologic and taxonomic analysis of the weedy and cultivated Amaranthus hybridus species complex. Systematic Botany 40: 604-610.

DOI: https://doi.org/10.1600/036364415X688376

Agudelo-H CA. 2008. Amaranthaceae. Flora de Colombia No. 
23. Instituto de Ciencias Naturales, Universidad Nacional de Colombia. Bogotá DC. Colombia. ISSN 0120-4351

Akeroyd JR. 1993. Amaranthus L. In: Tutin TG, Burges NA, Chater AO, Edmondson JR, Heywood VH, Moore DM, Valentine DH, Walters SM, Webb DA (eds.).Flora Europaea (2 ed.) Vol.1: Psilotaceae to Platanaceae. 130-132. Cambridge University Press, Cambridge. ISBN 978-0-521-15366-9

Bojian B, Clemants SE, Borsch T. 2003. Amaranthus L. In: Wu ZY, Raven PH. \& Hong DY. (eds.), Flora of China 5: Umbellaceae-Basellaceae. 415-429. Science Press, Beijing and Missouri Botanical Garden Press, St. Louis. ISBN 9781930723276

Bravo W, Leon RG, Ferrell JA, Mulvaney MJ, Wood CW. 2017. Differentiation of life-history traits among Palmer amaranth populations (Amaranthus palmeri) and its relation to cropping systems and glyphosate sensitivity. Weed Science $\mathbf{6 5}$ : 339-349. DOI: https://doi.org/10.1017/wsc.2017.14

Bravo W, Leon RG, Ferrell JA, Mulvaney MJ, Wood CW. 2018. Evolutionary adaptations of Palmer amaranth (Amaranthus palmeri) to nitrogen fertilization and crop rotation history affect morphology and nutrient-use efficiency. Weed Science 66: 180-189. DOI: https://doi.org/10.1017/wsc.2017.73

Carmona-Pinto W, Orsini-Velásquez G. 2010. Sinopsis del subgénero Amaranthus (Amaranthus, Amaranthaceae) en Venezuela. Acta Botánica Venezuelica 33: 329-356.

Carretero JL. 1990. Amaranthus L. In: Castroviejo S, Laínz M, López G, Montserrat P, Muñoz F, Paiva J, Villar L (eds.). Flora Ibérica. Plantas vasculares de la Península Ibérica e Islas Baleares. 559-569. Real Jardín Botánico, CSIC, Madrid. ISBN 84-00-06221-3

Chandi A, Milla-Lewis SR, Jordan DL, York AC, Burton JD, Zuleta MC, Whitaker JR, Culpepper AS. 2013. Use of AFLP markers to assess genetic diversity in Palmer amaranth (Amaranthus palmeri) populations from North Carolina and Georgia. Weed Science 61: 136-145.

DOI: https://doi.org/10.1614/WS-D-12-00053.1

Costea M, Sanders A, Waines G. 2001. Preliminary results towards a revision of the Amaranthus hybridus complex (Amaranthaceae). Sida 19: 931-974.

Culpepper AS, Grey TL, Vencill WK, Kichler JM, Webster TM, Brown SM, York AC, Davis JW, Hanna WW. 2006. Glyphosate-resistant Palmer amaranth (Amaranthus palmeri) confirmed in Georgia. Weed Science 54: 620-626. DOI: https://doi.org/10.1614/WS-06-001R.1

Eliasson UH. 1987. Amaranthaceae. In: Harling G, Andersson L (eds.). Flora of Ecuador 28. 1-138. Göteborg University and Museum of Natural History, Stockholm. ISBN: 9188702235 , 9789188702234

Espitia E, Escobedo D, Mapes EC, Núñez CA. 2010. Áreas prioritarias para colectar germoplasma de Amaranthus en México con base en la diversidad y riqueza de especies. Revista Mexicana de Ciencias Agrícolas 1: 609-617.

Flores-Olvera H, Czaja A, Estrada-Rodríguez JL, Romero U. 2016. Floristic Diversity of Halophytic Plants of Mexico. In: Khan MA, Boër B, Özturk M, Clüsener-Godt M, Gul B. Breckle SW. Sabkha Ecosystems Volume V: The Americas. 299-327. Springer. ISBN 978-3-319-27093-7

Franssen AS, Skinner DZ, Al-Khatib K, Horak MJ, Kulakow PA.
2001. Interspecific hybridization and gene flow of ALS resistance in Amaranthus species. Weed Science 49: 598-606. DOI: https://doi.org/10.1614/0043-1745(2001)049[0598:IHAGFO ]2.0. $\mathrm{CO} ; 2$

Gaeddert JW, Peterson DE, Horak MJ. 1997. Control and crossresistance of an acetolactate synthase inhibitor-resistant Palmer amaranth (Amaranthus palmeri) biotype. Weed Technology 11: 132-137.

DOI: https://doi.org/10.1017/S0890037X00041464

Gaines TA, Shaner DL, Ward SM, Leach JE, Preston C, Westra P. 2011. Mechanism of resistance of evolved glyphosateresistant Palmer amaranth (Amaranthus palmeri). Journal of Agricultural and Food Chemistry 59: 29-38.

DOI: https://doi.org/10.1021/jf104719k

Gonçalves-Netto A, Nicolai M, Carvalho SJP, Borgato EA, Christoffoleti PJ. 2016. Multiple resistance of Amaranthus palmeri to ALS and EPSPS inhibiting herbicides in the state of Mato Grosso, Brazil. Planta Daninha 34: 581-587. DOI: http://dx.doi.org/10.1590/s0100-83582016340300019

González-Iturbe JA, Olmsted I, Tun-Dzul F. 2002. Tropical dry forest recovery after long term Henequen (sisal, Agave fourcroydes Lem.) plantation in northern Yucatan, Mexico. Forest Ecology and Management 167: 67-82. DOI: https://doi.org/10.1016/S0378-1127(01)00689-2

Gossett BJ, Murdock EC, Toler JE. 1992. Resistance of Palmer amaranth (Amaranthus palmeri) to the dinitroaniline herbicides. Weed Technology 6: 587-591.

Heap I. 2019. The International Survey of Herbicide Resistant Weeds. <www.weedscience.org > (accessed February 21, 2019).

Hernández-Ledesma P, Berendsohn WG, Borsch T, von Mering S, Akhani H, Arias S, Castañeda-Noa I, Eggli U, Eriksson R, Flores-Olvera H, Fuentes-Bazán S, Kadereit G, Klak C, Korotkova N, Nyffeler R, Ocampo R, Ochoterena H, Oxelman B, Rabeler RK, Sanchez A, Schlumpberger BO, Uotila P. 2015. A taxonomic backbone for the global synthesis of species diversity in the angiosperm order Caryophyllales. Willdenowia 45: 281-383.

DOI: https://doi.org/10.3372/wi.45.45301

Horak MJ, Peterson DE. 1995. Biotypes of Palmer amaranth (Amaranthus palmeri) and common waterhemp (Amaranthus rudis) are resistant to imazethapyr and thifensulfuron. Weed Technology 9: 192-195.

DOI: https://doi.org/10.1017/S0890037X00023174

Iamonico D. 2012. Amaranthus powellii subsp. cacciatoi comb. et stat. nov. (Amaranthaceae). Nordic Journal of Botany 30: 1216. DOI: https://doi.org/10.1111/j.1756-1051.2011.01080.x

Iamonico D. 2015a. Amaranthaceae Juss. In: Euro+Med Plantbase - the information resource for Euro-Mediterranean plant diversity. $<$ http://euromed.luomus.fi/euromed_map.php?taxo $\mathrm{n}=449921$ \&size=medium $>$ (accessed February 22, 2019).

Iamonico D. 2015b. Taxonomic revision of the genus Amaranthus (Amaranthaceae) in Italy. Phytotaxa 199: 1-84. DOI: http://dx.doi.org/10.11646/phytotaxa.199.1.1

Iamonico D, El Mokni R. 2017. Amaranthus palmeri, a second record for Africa and notes on A. sonoriensis nom. nov. Bothlaia Africa Biodiversity \& Conservation 47: a2100. DOI: http://dx.doi.org/10.4102/abc.v47i1.2100

Iamonico D, El Mokni R. 2018. A new addition to the alien flora 
of Tunisia, Amaranthus spinosus L. (Amaranthaceae s.1.), with notes on A. diacanthus Raf. Acta Botanica Croatica DOI: https://doi.org/10.2478/botcro-2018-0009

INEGI [Instituto Nacional de Estadística y Geografía]. 2017a. Anuario estadístico y geográfico de Campeche 2017. Instituto Nacional de Estadística y Geografía. México.

INEGI. 2017b. Anuario estadístico y geográfico de Quintana Roo 2017. Instituto Nacional de Estadística y Geografía. México.

INEGI. 2017c. Anuario estadístico y geográfico de Yucatán 2017. Instituto Nacional de Estadística y Geografía. México.

Jhala AJ, Sandell LD, Rana N, Kruger GR, Knezevic SZ. 2014. Confirmation and control of triazine and 4-hydroxyphenylpyruvate dioxygenase-inhibiting herbicide-resistant Palmer amaranth (Amaranthus palmeri) in Nebraska. Weed Technology 28: 28-38.

DOI: https://doi.org/10.1614/WT-D-13-00090.1

Kauffman CS, Weber LE. 1990. Grain amaranth. In: Janick J \& Simon JE (eds.). Advances in new crops. 127-139. Timber Press, Portland. OR.

Klingaman TE, Oliver LR. 1994. Palmer amaranth (Amaranthus palmeri) interference in soybeans (Glycine max). Weed Science 42: 523-527.

DOI: https://doi.org/10.1017/S0043174500076888

Koo DH, Molin WT, Saski CA, Jiang J, Putta K, Jugulam M, Friebe B, Gill BS. 2018. Extrachromosomal circular DNAbased amplification and transmission of herbicide resistance in crop weed Amaranthus palmeri. Proceedings of the $\mathrm{Na}$ tional Academy of Sciences 115: 3332-3337.

DOI: https://doi.org/10.1073/pnas.1719354115

Massinga RA, Currie RS, Horak MJ, Boyer J. 2001. Interference of Palmer amaranth in corn. Weed Science 49: 202-208. DOI: https://doi.org/10.1614/0043-1745(2001)049[0202:IOPAIC] 2.0.CO;2

Morgan GD, Baumann PA, Chandler JM. 2001. Competitive impact of Palmer amaranth (Amaranthus palmeri) on cotton (Gossypium hirsutum) development and yield. Weed Technology 15: 408-412. DOI:

https://doi.org/10.1614/0890-037X(2001)015[0408:CIOPA A]2.0.CO;2

Morichetti S, Cantero JJ, Núñez C, Barboza GE, Espinar LA, Amucastegui A, Ferrell J. 2013. Sobre la presencia de Amaranthus palmeri (Amaranthaceae) en Argentina. Boletín de la Sociedad Argentina de Botánica 48: 347-354.

Mosyakin SL, Robertson KR. 1996. New infrageneric taxa and combinations in Amaranthus (Amaranthaceae). Annales Botanici Fennici 33: 275-281.

Mosyakin SL, Robertson KR. 2004. Amaranthus L. In: Flora of North America Editorial Committee (eds.). 1993+. Flora of North America North of Mexico. 20+ vols. 410-435. New York and Oxford. ISBN 978-0195173895

Müller-Schärer H, Schaffner U, Steinger T. 2004. Evolution in invasive plants: implications for biological control. Trends in Ecology and Evolution 19: 417-422.

DOI: https://doi.org/10.1016/j.tree.2004.05.010

Nakka S, Thompson CR, Peterson DE, Jugulam M. 2017. Target site-based and non-target site based resistance to ALS inhibitors in Palmer amaranth (Amaranthus palmeri). Weed Science 65: 681-689. DOI: https://doi.org/10.1017/wsc.2017.43
Neve P, Norsworthy JK, Smith KL, Zelaya IA. 2011. Modeling glyphosate resistance management strategies for Palmer amaranth (Amaranthus palmeri) in cotton. Weed Technology 25: 335-343. DOI: https://doi.org/10.1614/WT-D-10-00171.1

Norsworthy JK, Griffith GM, Scott RC, Smith KL, Oliver LR. 2008. Confirmation and control of glyphosate-resistant Palmer amaranth (Amaranthus palmeri) in Arkansas. Weed Technology 22: 108-113.

DOI: https://doi.org/10.1614/WT-07-128.1

Norsworthy JK, Griffith G, Griffin T, Bagavathiannan M, Gbur EE. 2014. In-field movement of glyphosate-resistant Palmer amaranth (Amaranthus palmeri) and its impact on cotton lint yield: evidence supporting a zero-threshold strategy. Weed Science 62: 237-249.

DOI: https://doi.org/10.1614/WS-D-13-00145.1

Ornelas-Uribe R. 1982. Contribución al conocimiento del género Amaranthus en el estado de Jalisco. Cuadernos de divulgación Jalisco, 9-31. Universidad de Guadalajara. México.

Pedersen TM. 2016. Amaranthaceae. In: Ramella L. Flora de Paraguay. Editions des Conservatoire et Jardin botaniques de la Ville de Genève.

Radosevich SR, Holt JS, Ghersa CM. 2007. Ecology of Weeds and Invasive Plants: Relationship to agriculture and natural resource management. New Jersey: John Wiley \& Sons. ISBN 978-0-471-76779-4

Rejmánek M. 2000. Invasive plants: approaches and predictions. Austral Ecology 25: 497-506.

DOI: https://doi.org/10.1046/j.1442-9993.2000.01080.x

Robertson KR. 1981. The genera of Amaranthaceae in the southeastern United States. Journal of the Arnold Arboretum 62: 267-314.

Rowland MW, Murray DS, Verhalen LM. 1999. Full-season Palmer amaranth (Amaranthus palmeri) interference with cotton (Gossypium hirsutum). Weed Science 47: 305-309. DOI: https://doi.org/10.1017/S0043174500091815

Ruzin ES. 1999. Plant microtechnique and microscopy. New York: Oxford University Press. ISBN: 0195089561

Rzedowski GC, Rzedowski J. 2005. Flora fanerogámica del Valle de México. Pátzcuaro, Michoacán, México. Instituto de Ecología A.C. y Comisión Nacional para el Conocimiento y Uso de la Biodiversidad.

Sánchez-del Pino I, Flores-Olvera H, Valdés J. 1999. La familia Amaranthaceae en la flora halófila y gipsófila de México. Anales del Instituto de Biología Universidad Nacional Autónoma de México, Serie Botánica 70: 29-135.

Sánchez-del Pino I, Espadas C, Pool R. 2013. Taxonomy and richness of nine genera of Amaranthaceae s.s. (Caryophyllales) in the Yucatan Peninsula Biotic Province. Phytotaxa 107: 1-74. DOI: http://dx.doi.org/10.11646/phytotaxa.107.1.1

Sauer JD.1950. The grain amaranths: A survey of their history and classification. Annals of the Missouri Botanical Garden 37: 561-632. DOI: https://doi.org/10.2307/2394403

Sauer JD. 1955. Revision of the dioecious amaranths. Madroño 13: 5-46.

Sauer JD. 1957. Recent migration and evolution of the dioecious amaranths. Evolution 11: 11-31.

DOI: https://doi.org/10.1111/j.1558-5646.1957.tb02872.x

Smith DT, Baker RV, Steele GL. 2000. Palmer Amaranth (Ama- 
ranthus palmeri) impacts on yield, harvesting, and ginning in dryland cotton (Gossypium hirsutum). Weed Technology 14:122-126. DOI:

https://doi.org/101614/0890-037X(2000)014[0122:PAAPIO ]2.0. $\mathrm{CO} ; 2$

Solís-Fernández KZ. 2017. Valoración morfológica, usos y distribución del amaranto (Amaranthus cruentus L. y Amaranthus hybridus L.) en algunas zonas de la región maya. BSc Thesis. Instituto Tecnológico de Conkal.

Standley PC. 1917. Amaranthaceae. North American Flora 21: 95-169.

Standley PC. 1930. Flora of Yucatan. Field Museum of Natural History. Botanical Series 3:157-942.

DOI: https://doi.org/10.5962/bhl.title.2354

Standley PC, Steyermark JA. 1946. Amaranthaceae. In: Standley, PC, Steyermark JA, eds. Flora of Guatemala - Part IV. Fieldiana, Botany 24: 143-174.

Thiers B. 2019+. Index herbariorum, a global directory of public herbaria and associated staff. New York Botanical

Editor de sección: Monserrat Vázquez Sánchez

Contribución de los autores: ISP: colectó y determinó el material, escribió el artículo. ADE: colectó y fotografió el material en campo. AIM: colectó, midió y fotografió las estructuras descritas, escribió el artículo.
Garden's Virtual Herbarium. < http://sweetgum.nybg.org/ih/> (accessed February 19, 2019).

Vibrans H (ed.). 2009. Malezas de México. <http://www.conabio.gob.mx/malezasdemexico/2inicio/home-malezas-mexico.htm> (accessed February 19, 2019).

Villaseñor JL, Espinosa-García FJ. 1998. Catálogo de malezas de México. Universidad Nacional Autónoma de México. Fondo de Cultura Económica: Ciudad de México. ISBN-10: 9681658787, ISBN-13: 978-9681658786

Villaseñor JL, Espinosa-García JF. 2004. The alien flowering plants of Mexico. Diversity and Distributions 10: 113-123. DOI: https://doi.org/10.1111/j.1366-9516.2004.00059.x

Ward SM, Webster TM, Steckel LE. 2013. Palmer amaranth (Amaranthus palmeri): A review. Weed Technology 27: 1227. DOI: https://doi.org/10.1614/WT-D-12-00113.1

Wassom JJ, Tranel PJ. 2005. Amplified fragment length polymorphism-based genetic relationships among weedy Amaranthus species. Journal of Heredity 96: 410-416. DOI: https://doi.org/10.1093/jhered/esi065 
Apéndice 1. Información taxonómica de las especies de amarantos distribuidas en la Península de Yucatán.

Amaranthus arenicola I. M. Johnston. Nombres comunes (maya y español): no reportados. Hábitat: playas arenosas y dunas costeras (Sánchez-del Pino et al. 2013). Estatus migratorio en México: nativa (Flores-Olvera et al. 2016). Distribución en la Península de Yucatán: Yucatán (Sánchezdel Pino et al. 2013). Distribución general: EUA, México, se ha dispersado y ha sido introducido en otras regiones de Norteamérica y Europa (Sánchez-del Pino et al. 1999, Mosyakin \& Robertson 2004, Sánchez-del Pino et al. 2013).

Amaranthus australis (A. Gray) J. D. Sauer. Basónimo: Acnida australis A. Gray. Nombres comunes: Tesil, Xtexk ax (maya) y quelite, carricillo (español, Sánchez-del Pino et al. 2013).

Hábitat: Bosques tropicales, vegetación secundaria, en la rivera de los ríos y ruderal (Sánchez-del Pino et al. 2013). Estatus migratorio en México: Nativa (Mosyakin \& Robertson 2004). Distribución en la Península de Yucatán: Campeche, Quintana Roo y Yucatán (Sánchez-del Pino et al. 2013). Distribución general: Desde Florida hacia el sur a lo largo de la costa del Atlántico. EUA, México, las Islas Caribe, Colombia, Venezuela, Surinam y Brasil (Robertson 1981, Mosyakin \& Robertson 2004, Agudelo-H 2008).

Amaranthus crassipes Schlechtendal var. crassipes. Nombres comunes (maya y español): no reportados. Hábitat: Bosques tropicales y campos de cultivo (Sánchez-del Pino et al. 2013). Estatus migratorio en México: Introducida (Iamonico 2015b). Distribución en la Península de Yucatán: Campeche y Yucatán (Sánchez-del Pino et al. 2013). Distribución general: Estados Unidos, México, las Bahamas, las islas Caribe y el norte de Sudamérica, ocasionalmente es introducido a otras regiones (Robertson 1981, Mosyakin \& Robertson 2004, Agudelo-H 2008, Sánchez-del Pino et al. 2013).

Amaranthus cruentus L. Nombres comunes: quelite rojo, quelite morado (español; Ornelas-Uribe 1982). No reportados en maya. Hábitat: Cerca de cultivos y ruderal (Mosyakin \& Robertson 2004, Iamonico 2015b). Estatus migratorio en México: Nativa (Kauffman \& Weber 1990). Distribución en la Península de Yucatán: Campeche, Quintana Roo y Yucatán (Solís-Fernández 2017). Distribución general: cosmopolita, cultivada ampliamente (Bojian et al. 2003, Mosyakin \& Robertson 2004, Pedersen 2016).

Amaranthus dubius Martius ex Thellung. Nombres comunes: Zac-tez, Tes, macho, Chaca' atsam, Xtez, Ztez, Chakte Sil, Xties, Chac-tes, Tees kax, X-tez xiww, X-tees xiw, Xtes k'aax, Xteez (maya; Sánchez-del Pino et al. 2013); quelite (español; Ornelas-Uribe 1982). Hábitat: Bosque tropical, dunas costeras, manglar, campos de cultivo de henequén, vegetación secundaria y ruderal (Sánchez-del Pino et al. 2013). Estatus migratorio en México: Nativa (Eliasson 1987, Carmona-Pinto \& Orsini-Velásquez 2010). Distribución en la Península de Yucatán: Campeche, Quintana Roo y Yucatán (Sánchez-del Pino et al. 2013). Distribución general: EUA, México, las Indias Occidentales, Sudamérica. Introducido en Europa, Asia y África (Mosyakin \& Robertson 2004, Agudelo-H 2008).
Amaranthus greggii S. Watson. Nombres comunes: Xtez (maya, Standley 1930). No reportados en español. Sinónimos: Amaranthus annectens Blake. Hábitat: Dunas costeras, manglares, bosque tropical y vegetación secundaria (Sánchez-del Pino et al. 2013). Estatus migratorio en México: nativa (Flores-Olvera et al. 2016). Distribución en la Península de Yucatán: Campeche, Quintana Roo y Yucatán (Sánchez-del Pino et al. 2013). Distribución general: EUA y México, se distribuye sobre la línea costera desde la Península de Yucatán hasta Texas (Robertson 1981, Mosyakin \& Robertson 2004).

Amaranthus hybridus Linnaeus. Nombres comunes: Xtez (maya) y bledo, quelite (español; Sánchez-del Pino et al. 2013), quintonil (Rzedowski \& Rzedowski 2005). Habitat: bosques tropicales, dunas costeras, laderas, vegetación secundaria, ruderal y en la rivera de los ríos (Sánchez-del Pino et al. 2013). Estatus migratorio en México: Nativa (Mosyakin \& Robertson 2004, Vibrans 2009). Distribución en la Península de Yucatán: Campeche, Quintana Roo y Yucatán (Sánchez-del Pino et al. 2013). Distribución general: EUA, México, las Islas Caribe, Centroamérica, Sudamérica, ampliamente introducido en regiones tropicales, subtropicales y templadas-cálidas del Viejo Mundo (Eliasson 1987, Mosyakin \& Robertson 2004, Agudelo-H 2008).

Amaranthus palmeri S. Watson Nombres comunes: Alegría, bledo, bledo blanco, quelite, quintonil espinoso y quintonil (en español, Sánchez-del Pino et al. 1999). En maya no reportados. Hábitat: ruderal, en terrenos baldíos, bordes de canales de riego y drenaje, orillas de caminos y potreros (Vibrans 2009, Iamonico 2015b). Estatus migratorio en México: nativa (Vibrans 2009).

Distribución en la Península de Yucatán: Yucatán (probablemente Campeche, no confirmado).

Distribución general: EUA, México, introducida en Europa, Asia y Australia (Mosyakin \& Robertson 2004).

Amaranthus polygonoides Linnaeus. Nombres comunes: Zactez, Zacxtez, Sacxtez, Xacxtez (maya, Standley 1930) y quelite (español, Sánchez-del Pino et al. 1999). Sinónimos: Amaranthus berlandieri (Moquin-Tandon) Uline \& W.L. Bray. Hábitat: bosque tropical, vegetación secundaria y ruderal (Sánchez-del Pino et al. 2013). Estatus migratorio en México: introducida (Iamonico 2015b). Distribución en la Península de Yucatán: Yucatán (Sánchez-del Pino et al. 2013). Distribución general: EUA, México, el norte de Sudamérica, las Bahamas y las Islas Caribe, introducido en Europa (Robertson 1981, Mosyakin \& Robertson 2004, Iamonico 2015b).

Amaranthus powellii S. Watson. Nombres comunes: Tes Xiu, Tes-de monte alto (maya, Sánchez-del Pino et al. 2013) y quelite (español, Sánchez-del Pino et al. 1999). Hábitat: bosque tropical, vegetación secundaria, ruderal y campos de cultivo (Sánchez-del Pino et al. 2013). Estatus Migratorio en México: nativa (Mosyakin \& Robertson 2004). Distribución en la Península de Yucatán: Campeche y Yucatán (Sánchezdel Pino et al. 2013). Distribución general: EUA y México, introducido o naturalizado en Sudamérica, norte y centro de Europa, Asia y Australia (Mosyakin \& Robertson 2004, Vibrans 2009). 
Amaranthus spinosus Linnaeus. Nombres comunes: X-tes, Xtex, Te'si, Kix tees, X tez kax, X-tees xiw o tees, Xkis-xtes, X-tez (maya) y bledo, espino, quelite (español, Sánchez-del Pino et al. 2013); quelite espinoso (español, Ornelas-Uribe 1982). Hábitat: bosque tropical, campos de cultivo, dunas costeras, manglares, vegetación secundaria, ruderal y en la ribera de los ríos (Sánchez-del Pino et al. 2013). Estatus migratorio en México: nativa (Mosyakin \& Robertson 2004). Distribución en la Península de Yucatán: Campeche, Quintana Roo y Yucatán (Sánchez-del Pino et al. 2013). Distribución general: EUA, México, las Indias Occidentales, Centroamérica, Sudamérica, introducido en casi todo el mundo (Mosyakin \& Robertson 2004, Agudelo-H. 2008, Pedersen 2016).
Amaranthus viridis Linnaeus. Nombres comunes: Quelite o bledo (español, Sánchez-del Pino et al. 2013). No reportados en maya. Hábitat: bosque tropical, campos de cultivo, arvense ocasional, vegetación urbana y ruderal (Vibrans 2009, Sánchez-del Pino et al. 2013). Estatus migratorio en México: introducida (Mosyakin \& Robertson 2004, Villaseñor \& Espinosa-García 2004, Rzedowski \& Rzedowski 2005, Vibrans 2009, Iamonico 2015b). Distribución en la Península de Yucatán: Campeche y Yucatán (Sánchez-del Pino et al. 2013). Distribución general: es una especie pantropical que también se distribuye como maleza en regiones templadas (Eliasson 1987, Pedersen 2016). 
Apéndice 2. Literatura con ilustraciones disponibles del resto de especies de Amaranthus distribuidas en la Península de Yucatán.

Amaranthus arenicola: Planta femenina figura 15, página 54 (Sánchez-del Pino et al. 2013). Planta masculina figura 16, página 55 (Sánchez-del Pino et al. 2013).

Amaranthus australis: Figura 17, página 56 (Sánchez-del Pino et al. 2013).

Amaranthus crassipes var. crassipes: Figura 18, página 57 (Sánchez-del Pino et al. 2013).

Amaranthus cruentus: Figura 24, página 56 (Iamonico 2015b). Figura 24, página 80 (Pedersen 2016).

Amaranthus dubius: Figura 19, página 58 (Sánchez-del Pino et al. 2013).
Amaranthus greggii: Planta femenina figura 20, página 59 (Sánchez-del Pino et al. 2013). Planta masculina figura 21, página 60 (Sánchez-del Pino et al. 2013).

Amaranthus hybridus: Figura 22, página 61 (Sánchez-del Pino et al. 2013).

Amaranthus polygonoides: Figura 19, página 44 (Iamonico 2015b).

Amaranthus powelli: Figura 23, página 62 (Sánchez-del Pino et al. 2013).

Amaranthus spinosus: Figura 24, página 63 (Sánchez-del Pino et al. 2013).

Amaranthus viridis: Figura 25, página 64 (Sánchez-del Pino et al. 2013). 